\title{
Estimating the mass of cosmic rays by combining radio and muon measurements
}

\author{
Ewa M. Holt ${ }^{1,2, \star}$ for the Pierre Auger Collaboration ${ }^{3, \star \star}$ \\ ${ }^{1}$ Karlsruhe Institute of Technology (KIT), Institut für Kernphysik, Karlsruhe, Germany \\ ${ }^{2}$ Instituto de Tecnologías en Detección y Astropartículas (CNEA, CONICET, UNSAM), CABA, Argentina \\ ${ }^{3}$ Observatorio Pierre Auger, Av. San Martín Norte 304, 5613 Malargüe, Argentina
}

\begin{abstract}
The Auger Engineering Radio Array (AERA) is a radio detector at the Pierre Auger Observatory and it is dedicated to measure the radio emission of cosmic-ray air showers. AERA is co-located with the underground muon detectors of the Auger Muons and Infill for the Ground Array (AMIGA). This provides a perfect setup to experimentally test the benefits of combining muons and radio emission for estimating the primary mass. We have investigated this combination using air-shower simulations. We compared the performance for mass separation of this new method to alternative methods in which the electrons and muons are measured with particle detectors at the surface. For showers with zenith angles below $50^{\circ}$ the new method is of comparable performance, and for showers more inclined than $50^{\circ}$ it is clearly superior. Therefore, measuring the radio signal in addition to the muons significantly improves the mass sensitivity compared to techniques using solely particle measurements.
\end{abstract}

\section{Introduction}

To reveal the origin of high- and ultra-high energy cosmic rays, mass dependent measurements of the energy spectrum and arrival directions are of prime importance. Cosmic rays at these energies are measured via extensive air showers they induce in the atmosphere. The ratio of the muonic and electromagnetic component of these air showers is a well known mass estimator. The shower components can be measured with particle detectors at the ground or indirectly via the electromagnetic radiation produced from the showers in the atmosphere, such as radio emission. We have developed a novel technique to estimate the mass of cosmic rays by combining measurements of muons and the radio emission to estimate the mass of the primary cosmic rays.

At the Pierre Auger Observatory in Argentina [1], muons are measured separately by underground scintillators [2]. The radio emission is measured by AERA, consisting of a radio antenna array [3]. Both detectors are co-located and measure cosmic ray air showers above an energy of $10^{17.5} \mathrm{eV}$.

We investigated the power of the muon-radio combination to separate air showers induced by proton and iron nuclei, using CORSIKA simulations and the hadronic model QGSJETII-04. We studied the influence of the detector responses of AMIGA and AERA to the mass separation power and combined measurements of AMIGA and AERA for the first time. In this work, we give an overview of the most important results. For more details on the analyses, see Refs. [4] and [5].

\footnotetext{
$\star^{\star}$ e-mail: ewa.holt@kit.edu

${ }^{\star \star}$ Full author list at http://www.auger.org/archive/authors_2018_06.html
} 


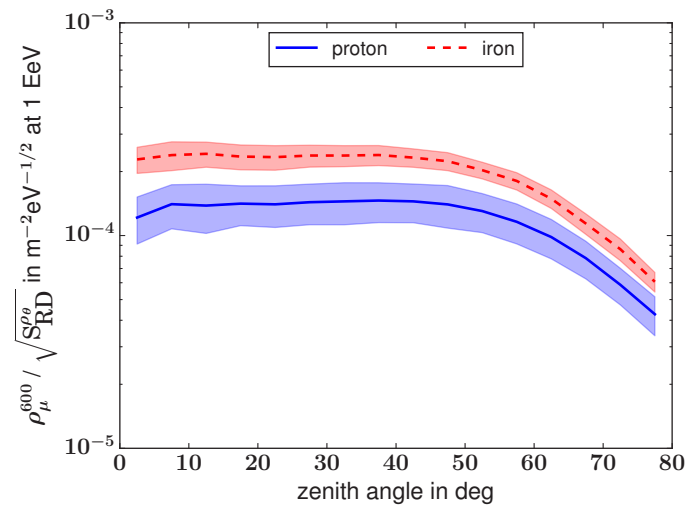

(a)

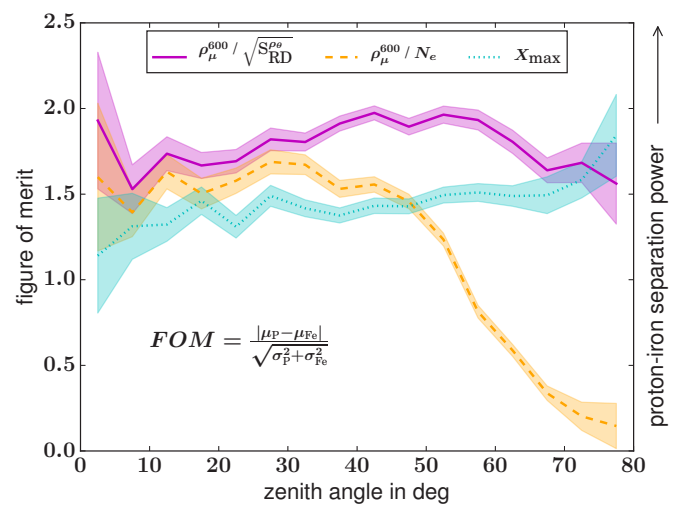

(b)

Figure 1. (a) Muon-radio mass estimator versus zenith angle for showers with a primary energy of 1 $\mathrm{EeV}$. The lines show the mean values $\mu_{\mathrm{i}}$ and the shaded areas depict the standard deviations $\sigma_{i}$. (b) Mass separation power of different mass estimators, represented by the figure of merit (FOM). The indices signify proton $(\mathrm{P})$ and iron nuclei $(\mathrm{Fe})$. No uncertainties from detector effects are included.

\section{Mass separation power}

The ratio of the muons and the radio emission, represented by observables as measured in AMIGA and AERA, is shown in Fig. 1a. The size of the muonic component is proportional to the muon density $\rho_{\mu}^{600}$ at a distance of $600 \mathrm{~m}$ to the shower axis, which is used here as the observable. The square root of the radiation energy $S_{\mathrm{RD}}^{\rho_{\theta}}$, which is the energy contained in the radio emission, represents an observable for the radio emission. Here, the observables are true values without any uncertainties arising from detector responses, background and reconstruction methods. The error band depicts the standard deviation due to shower-to-shower fluctuations. The ratio $\rho_{\mu}^{600} / \sqrt{ }\left(\mathrm{S}_{\mathrm{RD}}^{\rho_{\theta}}\right)$ is clearly separated for proton and iron nuclei for all zenith angles. It is constant until a zenith angle of $55^{\circ}$, which corresponds to the measurement range of AMIGA. It decreases for larger zenith angles, at which a fraction of the muons decay before reaching the ground.

Fig. 1b shows the mass separation power of the novel mass estimator in form of the figure of merit (FOM). In addition, the figure of merit is shown for the ratio of muons and electrons, and the shower maximum $X_{\max }$, which are classical mass estimators. At large zenith angles, the electromagnetic component is absorbed in the atmosphere, which diminished the mass separation power when combining muons and electrons. $X_{\max }$ is subject to shower-to-shower fluctuations, in particular for proton showers. On the contrary, the atmosphere is transparent for the radio emission and hence the emission is approximately constant at the ground for all zenith angles. In addition, it suffers less shower-to-shower fluctuations. Therefore, considering true observables without measurement uncertainties, the muon-radio combination shows a superior mass separation power compared to some classical estimators, in particular for large zenith angles.

\section{Application to AMIGA and AERA measurements}

We applied the novel mass estimator to coincident measurements of AMIGA and AERA. Therefore, we first studied the influence of the measurement uncertainties by adding the detector responses and measured radio background to the simulations. The reconstructed observables including the detectors are shown in Fig. 2a. The figure of merit is reduced by about 0.5 compared to the pure air-shower simulations due to measurement and reconstruction uncertainties. However, this is still sufficient for mass composition studies. 


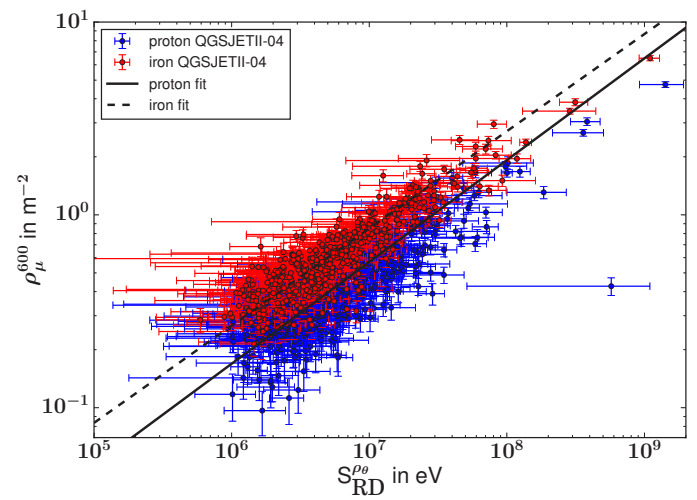

(a)

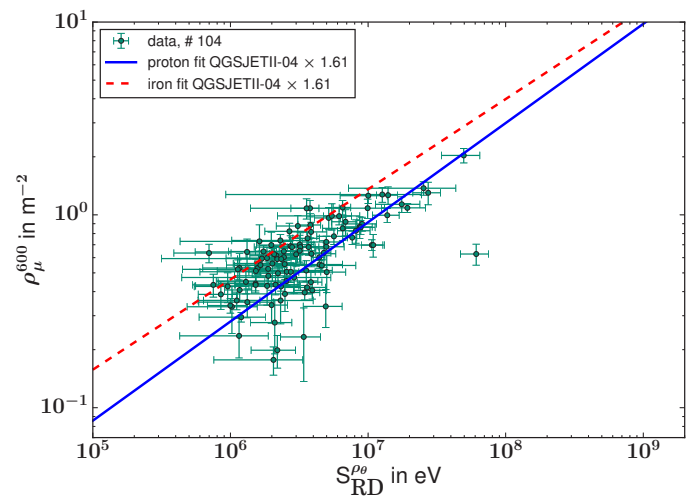

(b)

Figure 2. Correlation of the muon density and the radiation energy for (a) simulations including detector responses of AMIGA and AERA and measured radio background, and (b) coincident measurements of AMIGA and AERA.

We analyzed about one year of measurements of AMIGA and AERA and found 104 coincident events. The reconstructed muon density and radiation energy of these events are shown in Fig. $2 b$. For comparison, the fits for proton and iron from Fig. 2a are shown, shifted by a known discrepancy between the muon number predicted from QGSJETII-04 and measurements of $61 \%$ [6]. The average distribution of the events follows the simulated correlations and its magnitude lies within the mean of the simulations. This confirms the magnitude of the discrepancy of the muon number. More importantly, it demonstrates the potential of the novel mass estimator combining muon and radio measurements.

\section{Conclusion}

We developed a novel technique to estimate the mass of cosmic rays by combining measurements of muons and radio emission of air showers. Thereby, the muons are measured in form of the muon density $\rho_{\mu}^{600}$ at $600 \mathrm{~m}$ distance to the shower axis and the radio emission in form of the radiation energy $S_{\mathrm{RD}}^{\rho_{\theta}}$. Air-shower simulations (without detector responses) show that the mass estimator formed by these observables, $\rho_{\mu}^{600} / \sqrt{ }\left(\mathrm{S}_{\mathrm{RD}}^{\rho_{\theta}}\right)$, has a superior mass separation power compared to some classical mass estimators, in particular for inclined showers. We applied the novel technique to coincidence measurements of AMIGA and AERA and confirmed experimentally the correlation of the two observables. The novel technique can further be applied to other experiments combining muon and radio detectors, as already aimed for in various activities, building the Auger Radio Upgrade [7] or equipping IceTop with radio antennas [8].

\section{References}

[1] A. Aab et al. [Pierre Auger Collaboration], Nucl. Instrum. Meth. A798, 172-213 (2015)

[2] A. Aab et al. [Pierre Auger Collaboration], JINST 11, P02012 (2016)

[3] E. M. Holt for the Pierre Auger Collaboration, Proceedings of Science ICRC2017, 492

[4] E. M. Holt, PhD Thesis, KIT and UNSAM, 2018, DOI: 10.5445/IR/1000083318

[5] E. M. Holt, F. G. Schröder, in preparation for Eur. Phys. J. C (2018)

[6] A. Aab et al. [Pierre Auger Collaboration], Phys. Rev. Lett. 117, 192001 (2016)

[7] Hörandel, J. R. for the Pierre Auger Collaboration, Eur. Phys. J. WoC, this Issue

[8] A. Balagopal V. et al., Eur. Phys. J. C78.2, 111 (2018) 